\title{
CARATERIZAÇÃO MORFOMÉTRICA DOS FRUTOS DE PIMENTAS MALAGUETA E BIQUINHO
}

\author{
Haynna Fernandes Abud ${ }^{1}$, Roberto Fontes Araujo ${ }^{2}$, Cleide Maria Ferreira Pinto ${ }^{3}$, Eduardo Fontes
} Araujo ${ }^{4}$, Alisson Vinicius Araujo ${ }^{5}$, Jussara Aparecida dos Santos ${ }^{6}$

\begin{abstract}
RESUMO - Objetivou-se avaliar as características morfométricas dos frutos das pimentas Malagueta e Biquinho, de acordo com o estádio de maturação. Foram colhidos frutos aos 25, 40, 55, 70, 85 e 100 dias após a antese (DAA) e classificados quanto ao seu estádio fenológico com base na coloração do exocarpo. Em seguida, foram realizadas determinações morfométricas, mensurando-se a massa, o comprimento, o diâmetro, a espessura do pericarpo. Foram estimados os coeficientes de correlação de Pearson entre as quatro variáveis analisadas. A massa de matéria seca também foi determinada. Existe correlação positiva entre as variáveis morfométricas massa, comprimento, diâmetro e espessura do pericarpo de frutos para as duas espécies. Os frutos da pimentamalagueta apresentavam-se completamente verdes aos 25 e 40 DAA, enquanto os da pimenta-biquinho aos 25 DAA. As alterações na coloração do exocarpo, para a cor laranja, foi observada aos 40 DAA para a pimentabiquinho e aos 55 DAA para a pimenta-malagueta. Aos 70 e 85 DAA, o exocarpo apresentava a coloração vermelha, sendo intensificada aos 100 DAA. Durante a maturação dos frutos das pimentas Malagueta e Biquinho ocorrem acréscimos nos valores das variáveis morfométricas até determinada época de maturidade e, em seguida, são observadas reduções.
\end{abstract}

Palavras chave: Capsicum frutescens L., Capsicum chinense Jacq, morfometria, maturidade fisiológica.

\section{MORPHOMETRIC CHARACTERIZATION OF MALAGUETA AND BIQUINHO AT DIFFERENT STAGES OF MATURATION}

\begin{abstract}
This study aimed to evaluate the morphometric characteristicsof the fruits of malagueta and biquinho peppers, according to the stage of maturation. Fruits were collected at 25, 40, 55, 70, 85 and 100 days after anthesis (DAA) and classified according to their developmental stage based on the exocarp coloring. Posteriorly, morphometric determinations by measuring the mass, length, diameter and the thickness of the pericarp. The Pearson's coefficients correlation were estimated between the four variables analyzed. The dry mass was also determined. There is positive correlation between morphometric variables mass, length, diameter and thickness of the pericarp of fruits of both species. The fruits of malagueta were completely green at 25 and 40 DAA, while biquinho pepper at 25 DAA. Changes in the exocarp coloring, to the orange color, was observed at 40 DAA for biquinho pepper and at 55 DAA for malagueta pepper. The exocarp of fruits had red coloring at 70 and 85 DAA, being intensified at 100 DAA. During the fruits maturation of Malagueta and Biquinho peppers occurs increases in the values of morphometric variables until a certain timeof maturation and posteriorly, are observed reductions.
\end{abstract}

Keywords: Capsicum frutescens L,. Capsicum chinense Jacq, morphometry physiologic maturity.

\footnotetext{
${ }^{1}$ Eng. Agrônomo, D.Sc Fitotecnia, UFV.hfabud@gmail.com

${ }^{2}$ EPAMIG Sudeste, Caixa Postal 216, CEP 36570-000 Viçosa-MG. roberto.araujo@epamig.br

${ }^{3}$ Pesq. EMBRAPA/EPAMIG, EPAMIG SUDESTE Caixa Postal 216, CEP 36570-000 Viçosa-MG. cleide@epamig.br

${ }^{4}$ Prof. da Universidade Federal de Viçosa (UFV) efaraujo@ufv.br

${ }^{5}$ Eng. Agrônomo, D.Sc. Fitotecnia, UFV.

${ }^{6}$ Eng. Agrônomo, M.Sc. Fitotecnia, UFV.
} 


\section{INTRODUÇÃO}

As pimentas Capsicum apresentam expressiva importância econômica e social para o agronegócio mundial. Produtos de pimenta, pungentes e não pungentes, representam, em volume, uma das mais importantes commodities de tempero no mundo.

As pimentas constituem matéria-prima para extração de corantes, aromatizantes e oleorresinas, substâncias utilizadas em produtos alimentícios, por conferir sabor e aumentar a estabilidade oxidativa dos lipídios. Têm altos valores vitamínicos além de ser fonte de antioxidantes naturais como a vitamina $\mathrm{C}$, os carotenóides, os quais têm atividade provitamina $\mathrm{A}$, vitamina $\mathrm{E}$, vitaminas do complexo B, compostos fenólicos, carboidratos, lipídeos, proteínas, fibras e sais minerais (Bosland \& Votava, 2000; Lutz \& Freitas, 2008; Pinto et al., 2013; Pereira et al., 2016; Maurya et al., 2017).

Os dados mundiais de produção de Capsicum, quando disponíveis, englobam pimentas e pimentões. Em 2014, a produção mundial de pimentas e pimentões frescos, foi cerca de 32,3 milhões de toneladas numa área cultivada de 1,9 milhões de hectares. $\mathrm{O}$ maior produtor foi a China com $50 \%$ do total produzido, seguido pelo México $(8,5 \%)$, Turquia $(6,6 \%)$, Indonésia $(5,8 \%)$ e Espanha (3,5\%) (Faostat, 2016). No Brasil, o agronegócio de pimentas Capsicum está entre os melhores exemplos de integração entre todos que atuam na cadeia produtiva dessa hortaliça. Minas Gerais é principal Estado produtor de pimentas, com produção de aproximadamente 2 toneladas em 2015 (Emater-MG, 2016).

A variabilidade genética no gênero Capsicum é consideravelmente elevada, visível principalmente nos frutos, que apresentam como características diferentes tamanhos, formatos, pungência (ardume) e coloração. Além da coloração do exocarpo, a firmeza dos frutos, o teor de sólidos solúveis, o teor de ácidos, a concentração de compostos voláteis, assim como as alterações em outros compostos químicos têm sido utilizados como indicadores de maturidade para vários produtos comerciais, inclusive a pimenta (Tadesse et al., 2002). Esses indicadores são utilizados para determinar a época de colheita apropriada, de modo que seus atributos de qualidade física e fisiológica se mantenham por longos períodos durante o armazenamento e a comercialização.
O estádio de maturação é um fator relevante quanto aos atributos de qualidade, pois influencia diretamente a composição dos frutos, uma vez que, durante o amadurecimento, ocorrem várias modificações bioquímicas, fisiológicas e estruturais. No caso de frutos de pimenta, o conhecimento prático sobre a maturação dos frutos e sementes, bem como do período ideal de colheita, assume grande importância agrícola, tendo em vista que plantas deste gênero apresentam florescimento contínuo, em que frutos de diferentes estádios de maturação estão presentes na mesma planta (Nascimento et al., 2006; Vidigal et al., 2009). Desse modo, a coloração dos frutos de pimenta é uma característica que pode ser utilizada como um indicativo da maturidade da semente, podendo ou não coincidir com o máximo de germinação e vigor (Vidigal et al., 2011).

A caracterização morfométrica de frutos tem sido bastante relatada como fundamental na identificação e caracterização botânica de espécies, na compreensão de desenvolvimento, além de subsidiar informações relevantes ao melhoramento vegetal das espécies. Diversas pesquisas na área de melhoramento vegetal apresentam como objetivo quantificar a divergência fenotípica entre acessos de Capsicum spp., com base na caracterização morfológica e agronômica, por meio de descritores quantitativos (peso, comprimento, diâmetro, número de sementes, espessura do pericarpo) e qualitativos (cor, pungência, formato, textura do fruto).

Bento et al. (2007) verificaram que o comprimento de frutos do gênero Capsicum variou de 10,24 a 143 mm e afirmam que, em função desta variável, podese determinar a forma de uso mais adequada para o acesso. Os frutos pequenos apresentaram maior potencial de utilização em molhos e os frutos maiores comercializados, preferencialmente, in natura ou desidratados. Sudré et al. (2005) observaram que as características que apresentam maior importância para a diversidade genética entre os acessos de Capsicum spp. foram o peso, o comprimento e o diâmetro dos frutos, além do peso de mil sementes. Contudo, existem poucas informações sobre a fisiologia de crescimento e maturação dos frutos de pimenta como relatado por Vidigal et al. (2009), Rêgo et al. (2011), Queiroz et al. (2011), Vidigal et al. (2011), entre outros. Diante do exposto, objetivou-se avaliar as características morfométricas dos frutos das pimentas malagueta e 
biquinho, de acordo com o estádio de maturação dos frutos, além de determinar a correlação entre estas características.

\section{MATERIAL E MÉTODOS}

Foram utilizadas sementes de pimenta-malagueta (C. frutescens L.) e de pimenta-biquinho (C. chinense Jacq.). As sementes foram produzidas na casa de vegetação da Empresa de Pesquisa Agropecuária de Minas Gerais (EPAMIG), a partir de sementes adquiridas por doação da Embrapa Hortaliças.

Realizou-se a semeadura em bandejas de isopor de 128 células, contendo substrato comercial utilizado para produção de olerícolas (Plantmax ${ }^{\circledR}$ ), em casa de vegetação, sendo os tratos culturais realizados conforme recomendações técnicas para a cultura. Quarenta dias após a semeadura em bandejas, quando as plantas apresentavam de três a cinco pares de folhas, foi realizado o transplantio das mudas para vasos de polietileno, com volume de cinco litros de substrato (Plantmax ${ }^{\circledR}$ ), distribuídos em casa de vegetação previamente limpa. Para garantir a pureza genética das sementes, evitando a ocorrência de polinização cruzada, foram colocados tecidos organza de coloração branca ao longo da casa de vegetação, separando as diferentes espécies.

Durante o florescimento, as flores foram etiquetadas diariamente, a partir da antese até ser obtido número de sementes suficientes para os testes propostos. Foram coletados frutos aos 25, 40, 55, 70, 85 e 100 dias após a antese (DAA), de acordo com a Figura 1. Após a colheita, os frutos das duas espécies de pimenta foram transportados ao Laboratório de Análises de Sementes e, classificados quanto ao seu estádio fenológico com base na coloração externa do exocarpo.

Para a determinação morfométrica dos frutos das pimentas malagueta e biquinho, utilizou-se uma amostra de 30 frutos, exceto para a variável massa de matéria seca. Foram mensurados:

Massa - os frutos foram pesados individualmente em balança digital (precisão $0,001 \mathrm{~g}$ ), determinandose a massa médio de frutos em grama/fruto;

Comprimento - mensurado da região compreendida entre o ápice e a base dos frutos utilizandose paquímetro digital, com resolução de $0,01 \mathrm{~mm}$ e os resultados foram expressos em $\mathrm{mm} /$ fruto;
Diâmetro - mensurado na região central dos frutos utilizando-se paquímetro digital, com resolução de $0,01 \mathrm{~mm}$ e os resultados foram expressos em $\mathrm{mm} /$ fruto;

Espessura do pericarpo - mensurada na região central dos frutos, utilizando-se paquímetro digital, com resolução de $0,01 \mathrm{~mm}$ e os resultados foram expressos em mm/fruto;

Massa de matéria seca dos frutos - os frutos foram colocados para secar em estufa de circulação forçada de ar, à temperatura de $65^{\circ} \mathrm{C}$, por 36 horas. Foram utilizados quatro repetições de 10 frutos para cada época de maturação e os resultados expressos em g/fruto.

O experimento foi realizado em delineamento inteiramente casualizado, com um número amostral de 30 frutos, em que os resultados obtidos foram submetidos à análise de variância utilizando-se o procedimento MIXED (SAS 9.3). Foi realizada decomposição ortogonal do fator tratamentos (dias após a antese - DAA) em efeitos linear, quadrático e cúbico. Ao se observar significância em nível de 5\% de probabilidade para o erro tipo I para algum dos efeitos, ajustou-se equação de regressão utilizando-se a declaração SOLUTION. Foram estimados os coeficientes de correlação de Pearson entre as variáveis massa, comprimento, diâmetro e espessura do pericarpo.

\section{RESULTADOS E DISCUSSÃO}

Na Figura 1, observa-se o aspecto visual dos frutos de pimenta-malagueta e de pimenta-biquinho em diferentes estádios de maturação aos 25, 40, 55, 70, 85 e 100 dias após a antese (DAA). Nas duas espécies, observou-se que aos 25 DAA os frutos apresentavamse completamente verdes. Com a degradação da clorofila e acúmulo de outros pigmentos como carotenóides e antocianinas (Taiz \& Zeiger, 2009) verificaram-se que para a pimenta-biquinho, a partir de $40 \mathrm{DAA}$ e, para a pimenta-malagueta, a partir dos $55 \mathrm{DAA}$, ocorrem alterações na coloração do exocarpo dos frutos para a cor laranja. Aos 70 e 85 DAA o exocarpo dos frutos das pimentas Malagueta e Biquinho apresentavam a coloração vermelha, sendo bastante intensificada aos 100 DAA.

É importante ressaltar que durante o amadurecimento de frutos ocorrem várias modificações bioquímicas, 

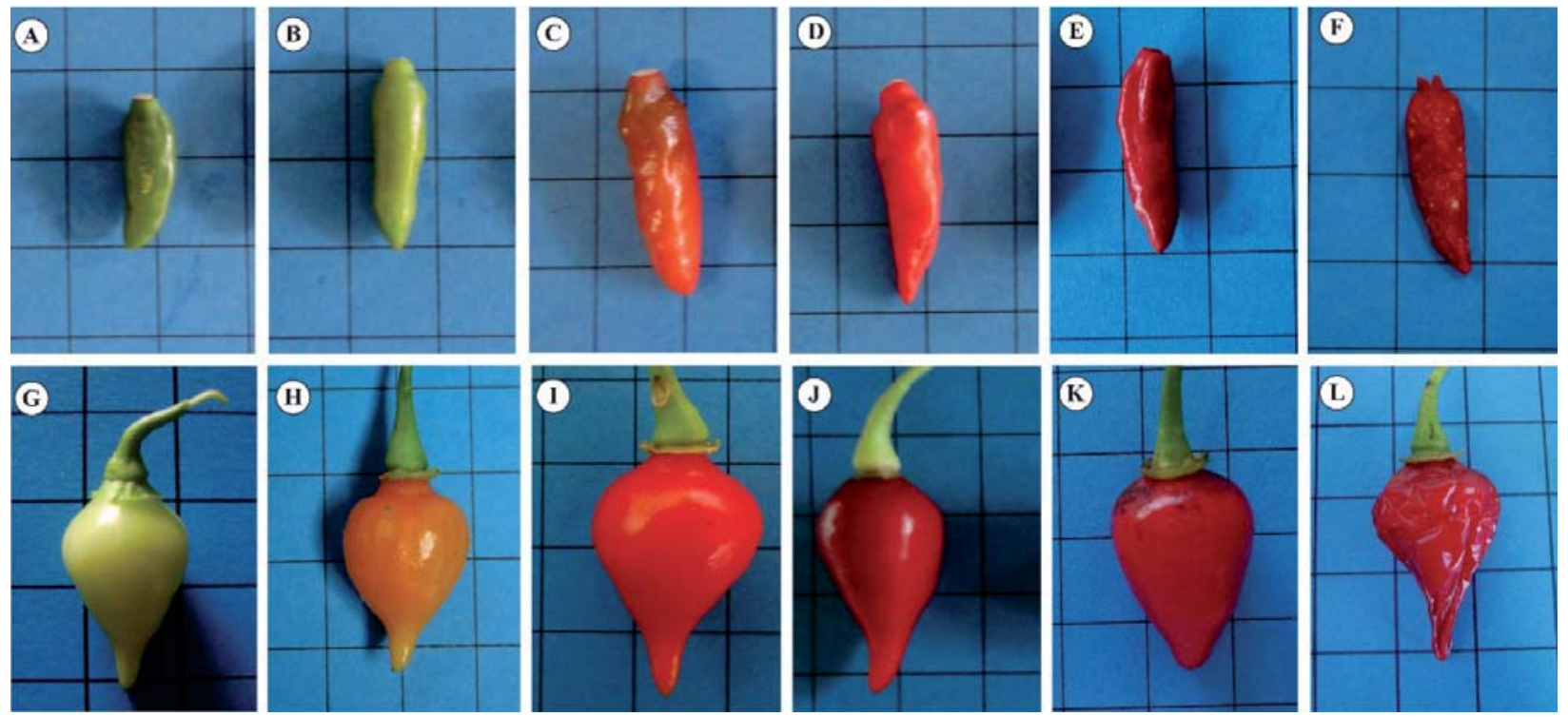

Figura 1 - Aspecto visual dos frutos de pimenta-malagueta (A - 25 DAA; B - 40 DAA; C - 55 DAA; D - 70 DAA; E - 85 DAA; F - 100 DAA) e biquinho (G - 25 DAA; H - 40 DAA; I - 55 DAA; J - 70 DAA; K - 85 DAA; L - 100 DAA) durante o processo de maturação.

fisiológicas e estruturais que determinam os padrões de qualidade. Menichini et al. (2009) verificaram diferenças na composição de frutos imaturos e maduros de $C$. chinense durante os estádios de desenvolvimento, com aumento na concentração de carotenoides e redução na concentração de fenóis, quando as pimentas atingiram a maturidade fisiológica.

De acordo com a Figura 1, é possível visualizar uma elevada alteração na turgescência dos frutos aos 100 DAA, principalmente para o "Biquinho". A perda de água pela transpiração e perda de matéria seca, devido ao aumento da atividade respiratória, refletem em perda de massa fresca total pós-colheita. Além das alterações de coloração e turgescência, Pino et al. (2006) afirmam que ocorrem alterações nos compostos voláteis responsáveis pelo aroma dos frutos verdes-maduros e maduros de C. chinense, que conferem aroma distinto para cada estádio, durante o amadurecimento dos frutos. Os autores identificaram 102 compostos diferentes, com predominância de diferentes tipos de álcoois, aldeídos e cetonas.

Na Tabela 1, está inserida a estatística descritiva referente à morfometria dos frutos de pimenta-malagueta e pimenta-biquinho. Dentre as variáveis analisadas, o diâmetro dos frutos apresentou a menor variação para a "Malagueta" e, o comprimento para a "Biquinho".
Para as duas espécies, as variáveis massa e espessura dos frutos apresentaram as maiores dispersões relativas, como denotam os seus coeficientes de variação.

Estudos relacionados à caracterização morfológica de frutos, sementes e plântulas possibilitam o entendimento dos métodos de propagação, assim como a análise de crescimento vegetativo, além de fornecer informações relativas à identificação de espécies, fornecendo subsídios para a diferenciação entre espécies, variedades e caracterização de aspectos ecológicos da planta, como dispersão e estabelecimento de plântulas. Outros autores também destacam a importância da caracterização morfológica de acessos deste gênero (Geleta et al., 2005; Oyama et al., 2006; Fonseca et al., 2008; Rêgo et al., 2009; Thul et al., 2009; Rêgo et al., 2011; Domenico et al., 2012; Junior e Silva et al., 2013).

$\mathrm{Na}$ Tabela 2, é apresentada a associação existente entre as variáveis massa, comprimento, diâmetro e espessura do pericarpo dos frutos das pimentas Malagueta e Biquinho, verificada por meio da correlação de Pearson. Para a "Malagueta", verificou-se elevada correlação entre as variáveis comprimento e diâmetro $(0,904)$, massa e espessura do pericarpo dos frutos $(0,835)$ e massa e diâmetro $(0,781)$. A correlação entre todas as variáveis analisadas para esta espécie foi 
Tabela 1 - Características físicas dos frutos de pimenta-malagueta (MA) e de pimenta-biquinho (BQ)

\begin{tabular}{cccccc}
\hline Espécie & Variável & Média & Desvio Padrão & Intervalo de Variação & CV (\%) \\
\hline \multirow{4}{*}{ MA } & Massa $(\mathrm{g})$ & 0,366 & 0,156 & $0,076-0,778$ & 42,7126 \\
& Comprimento $(\mathrm{mm})$ & 23,314 & 4,306 & $14,600-36,320$ & 18,4693 \\
& Diâmetro $(\mathrm{mm})$ & 5,820 & 0,637 & $4,380-7,610$ & 10,945 \\
& Espessura do Pericarpo $(\mathrm{mm})$ & 0,559 & 0,186 & $0,250-1,00$ & 33,2737 \\
& Massa $(\mathrm{g})$ & 1,101 & 0,313 & $0,454-1,893$ & 28,4287 \\
BQ & Comprimento $(\mathrm{mm})$ & 21,053 & 2,270 & $15,090-28,720$ & 10,7822 \\
& Diâmetro $(\mathrm{mm})$ & 12,156 & 1,641 & $7,760-16,880$ & 13,4995 \\
& Espessura do Pericarpo $(\mathrm{mm})$ & 1,746 & 0,395 & $0,970-2,620$ & 22,6231 \\
\hline
\end{tabular}

Tabela 2 - Correlação de Pearson referente às variáveis biométricas de frutos de pimenta-malagueta e de pimentabiquinho $(\mathrm{P}<0,001)$

\begin{tabular}{ccc}
\hline Espécie & Comparações & Correlação de Pearson \\
\hline MA & Massa x comprimento & 0,73054 \\
& Massa x diâmetro & 0,78147 \\
& Massa x espessura do pericarpo & 0,83527 \\
& Comprimento x diâmetro & 0,90414 \\
BQ & Comprimento x espessura do pericarpo & 0,44776 \\
& Massa x comprimento & 0,67904 \\
& Massa x diâmetro & 0,96648 \\
& Massa x espessura do pericarpo & 0,92362 \\
& Comprimento x diâmetro & 0,66306 \\
\end{tabular}

superior a 50\%, com exceção para a correlação existente entre o comprimento e a espessura do pericarpo dos frutos, que foi de 0,447. Para a "Biquinho", observouse que, todas as correlações foram acima de $50 \%$, sendo as mais elevadas associações verificadas entre as variáveis massa e diâmetro $(0,966)$ e massa e espessura do pericarpo dos frutos $(0,923)$.

As correlações positivas entre comprimento e diâmetro, massa e diâmetro, massa e espessura do pericarpo dos frutos, para ambas as espécies, são favoráveis ao rendimento de polpa dos frutos. Esta é uma característica que agrega valores econômicos a estas espécies, uma vez que a polpa dos frutos de pimenta apresenta relevante importância, sendo utilizada tanto in natura como industrializada na forma de condimentos, molhos, páprica, entre outros.

Em pesquisa semelhante, Tadesse et al. (2002) citam que o peso de frutos fresco de pimentão cv. Domino foi positivamente correlacionado a atributos físicos de crescimento como diâmetro $(0,93)$, comprimento $(0,81)$ e volume $(0,99)$, com $P \geq 0,001$. Lannes et al. (2007), em pesquisa com acessos da espécie $C$. chinense do banco de germoplasma da Universidade Federal de Viçosa (UFV), verificaram elevada correlação entre as variáveis peso e espessura do pericarpo $(\mathrm{r}=0,79)$, em que acessos com peso médio de $0,99 \mathrm{~g}$ apresentaram pericarpo de aproximadamente $1,57 \mathrm{~mm}$, enquanto acessos que apresentavam 19,15 g tinham pericarpo de 3,39 mm de espessura.

A massa dos frutos das pimentas Malagueta e Biquinho apresentaram comportamento quadrático (Figura 2). Aos 25 DAA observou-se massa de $0,352 \mathrm{~g}$ para a pimenta malagueta e $0,834 \mathrm{~g}$ para a pimenta biquinho. Os máximos valores de massa dos frutos foram de aproximadamente $0,458 \mathrm{~g}$ aos 54 DAA (Figura 2A) e 1,292 g aos 65 DAA (Figura 2B) para as pimentas Malagueta e Biquinho, respectivamente. Verificou-se que até os 54 e 65 DAA para as espécies, ocorreram incrementos na massa dos frutos das duas espécies e, a partir destas épocas de maturação, foram observados decréscimos dos seus valores, sendo que aos 100 DAA os frutos apresentaram $0,184 \mathrm{~g} \mathrm{e} 0,945 \mathrm{~g}$, respectivamente.

Vidigal et al. (2011) verificaram que para a pimenta cv. 'Amarela comprida' os frutos apresentaram aumento 
significativo até os 45 DAA, onde a partir desta época de maturação o peso dos frutos manteve-se constante até os 75 DAA.

O comprimento dos frutos das duas espécies apresentou comportamento matemático distinto de desenvolvimento durante a maturação (Figura 3). Para a pimenta-malagueta foi ajustado um comportamento cúbico, sendo que aos $25 \mathrm{DAA}$ os frutos apresentavam aproximadamente $22,12 \mathrm{~mm}$ de comprimento. $\mathrm{O}$ valor máximo foi verificado aos 79 DAA, quando os frutos apresentavam 26,69 $\mathrm{mm}$. A partir desta época houve reduções, sendo que aos $100 \mathrm{DAA}$, o comprimento dos frutos foi de aproximadamente $21,46 \mathrm{~mm}$ (Figura $3 \mathrm{~A}$ ). A pimenta-biquinho apresentou efeito linear para a variável comprimento de frutos. Aos $25 \mathrm{DAA}$ os frutos apresentaram comprimento médio de 20,12 $\mathrm{mm}$. De acordo com a Figura 3B é possível afirmar que houve acréscimos de comprimento a uma taxa de $0,02463 \mathrm{~mm}$ ao dia, sendo que aos 100 DAA foram observados frutos com aproximadamente $21,976 \mathrm{~mm}$ de comprimento.

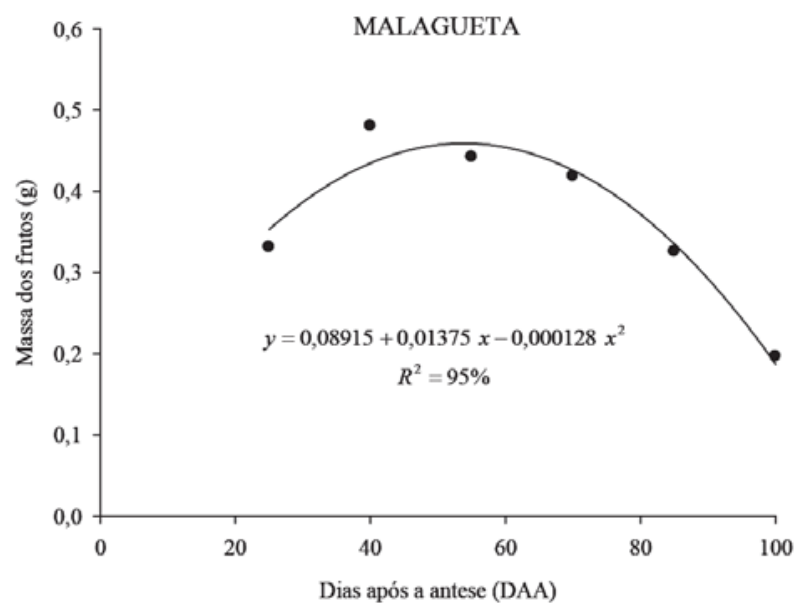

A

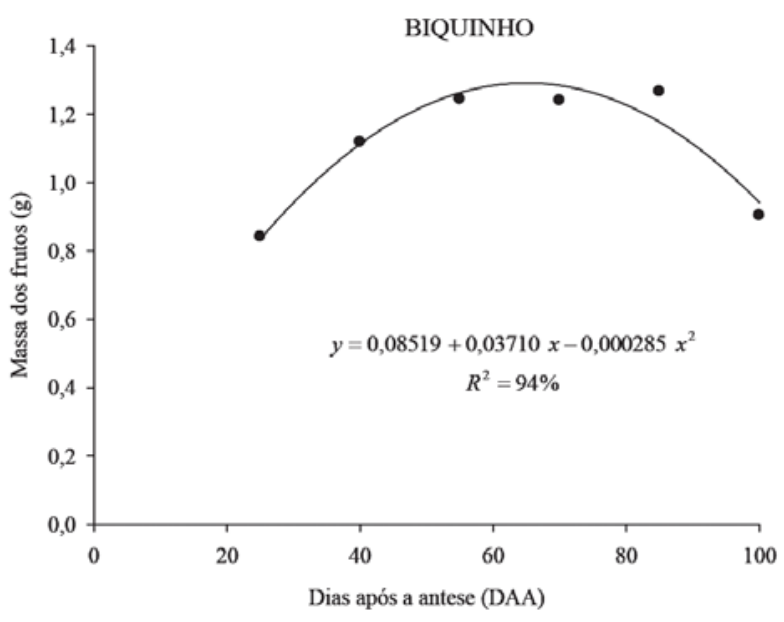

B

Figura 2 - Massa dos frutos de pimenta-malagueta (A) e de pimenta-biquinho (B) em diferentes estádios de maturação.

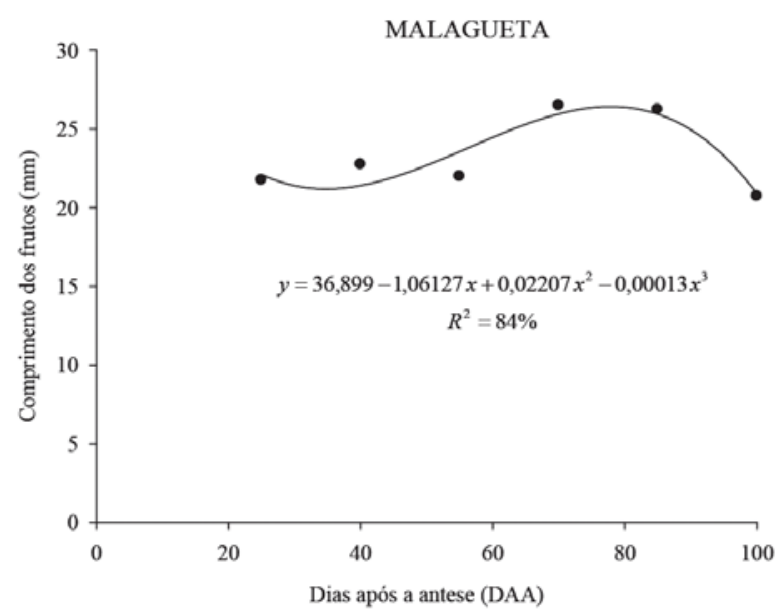

A

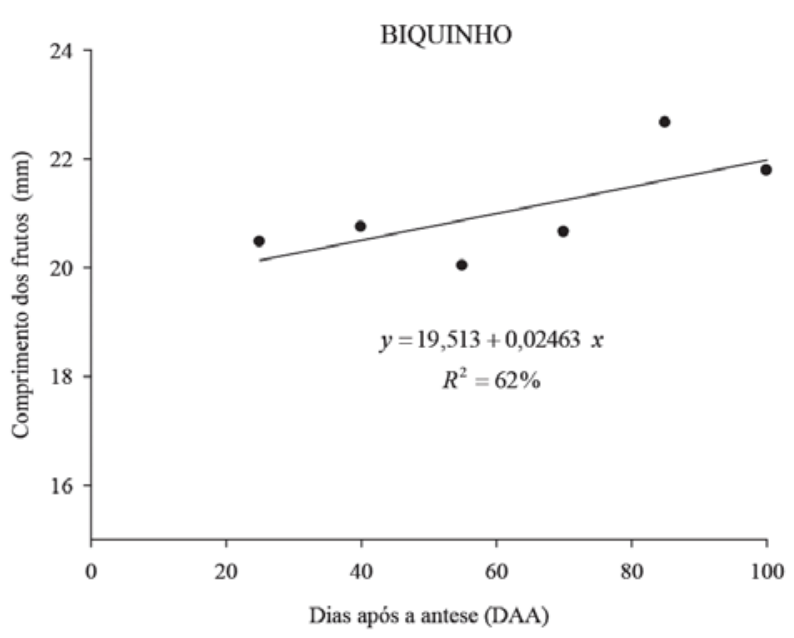

B

Figura 3 - Comprimento dos frutos de pimenta-malagueta (A) e de pimenta-biquinho (B) em diferentes estádios de maturação. 
$\mathrm{Na}$ Figura 4, visualiza-se que as duas espécies apresentaram comportamento quadrático para a variável diâmetro durante o crescimento e desenvolvimento dos frutos. Aos 25 DAA foi verificado diâmetro de 5,432 mm. A "Malagueta" apresentou diâmetro máximo aos 67 DAA com 6,05 mm. A partir desta época de maturação dos frutos, houve redução nos valores desta variável, sendo que aos 100 DAA os frutos apresentavam aproximadamente 5,67 mm (Figura 4A).

Para a "Biquinho" (Figura 4B), aos 25 DAA, os frutos apresentaram diâmetro médio de 10,58 mm, com acréscimos nos valores até 65 DAA, com aproximadamente $13,29 \mathrm{~mm}$, sendo este o maior valor. A partir desta época de maturação, foram verificados decréscimos dos valores, sendo que aos 100 DAA o diâmetro médio dos frutos foi de aproximadamente 11,28 $\mathrm{mm}$.

Assim como observado para a variável massa, para o diâmetro dos frutos também houve redução dos valores, que para as duas espécies ocorreu após os 60 DAA (Figura 4).

Na Figura 5 estão apresentados os valores referentes à espessura do pericarpo dos frutos de pimenta-malagueta e pimenta-biquinho colhidos em diferentes estádios de maturação. Para a pimenta-malagueta houve um comportamento cúbico. Aos 25 DAA verificou-se espessura média de aproximadamente $0,616 \mathrm{~mm}$. Até os 43 DAA foram observados incrementos nos valores

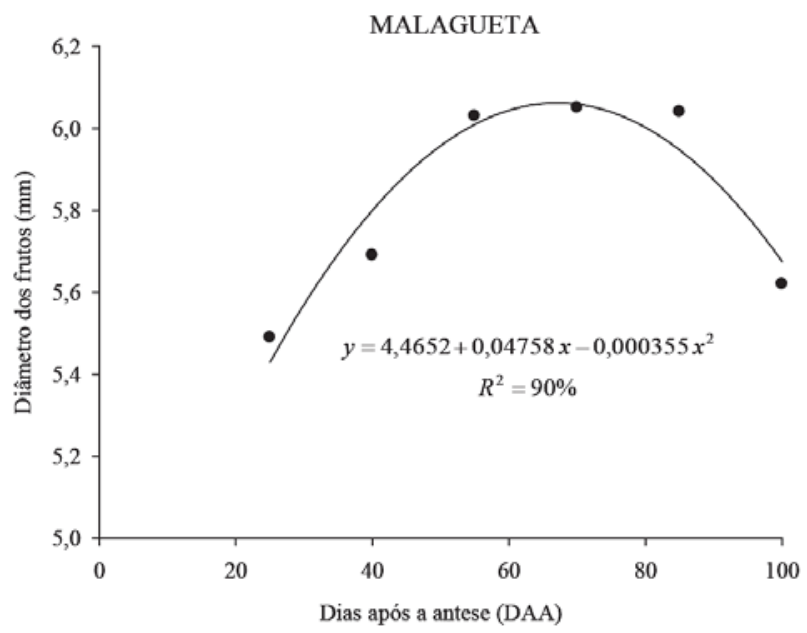

A referentes a esta variável, onde se verificou o valor máximo $0,704 \mathrm{~mm}$. A partir desta época de maturação ocorreram decréscimos nos valores, sendo que aos 100 DAA foi de $0,282 \mathrm{~mm}$.

Para a pimenta-biquinho, houve ajuste quadrático para a variável espessura do pericarpo dos frutos, em que aos 25 DAA foi de $1,30 \mathrm{~mm}$. Houve acréscimos na espessura do pericarpo até os $65 \mathrm{DAA}$, quando atingiu o seu valor máximo de $2,05 \mathrm{~mm}$ e a partir desta época de maturação, houve redução dos valores, que aos 100 DAA foi de $1,47 \mathrm{~mm}$.

A espessura do pericarpo é uma característica importante para variedades destinadas ao consumo in natura de frutos, tendo em vista que frutos com maior espessura de pericarpo são mais resistentes nos tratos pós-colheita. Além disso, esses frutos apresentam uma aparência mais apreciada pelos consumidores.

Lannes et al. (2007) citam que frutos menores de C. chinense apresentaram menor espessura de pericarpo e maior concentração de sólidos solúveis totais, sendo estes recomendados para a desidratação e produção de páprica. Enquanto os frutos maiores, independentemente de sua forma, apresentaram pericarpo mais espesso e, portanto, mais adequado ao consumo in natura.

A massa de matéria seca dos frutos apresentou efeito cúbico para as duas espécies (Figura 6). Aos 25 DAA, verificou-se $0,01686 \mathrm{~g} /$ fruto para a pimenta

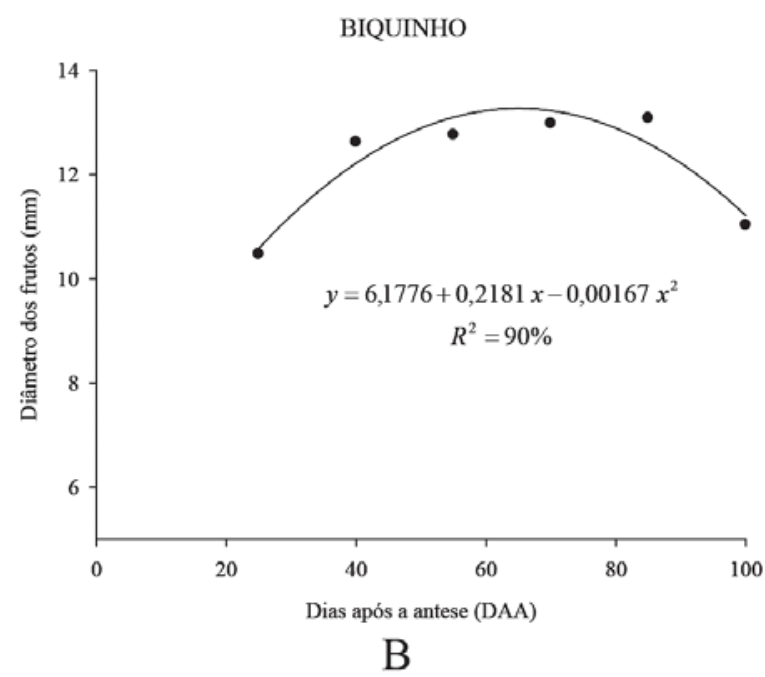

Figura 4 - Diâmetro dos frutos de pimenta-malagueta (A) e de pimenta-biquinho (B) em diferentes estádios de maturação. 


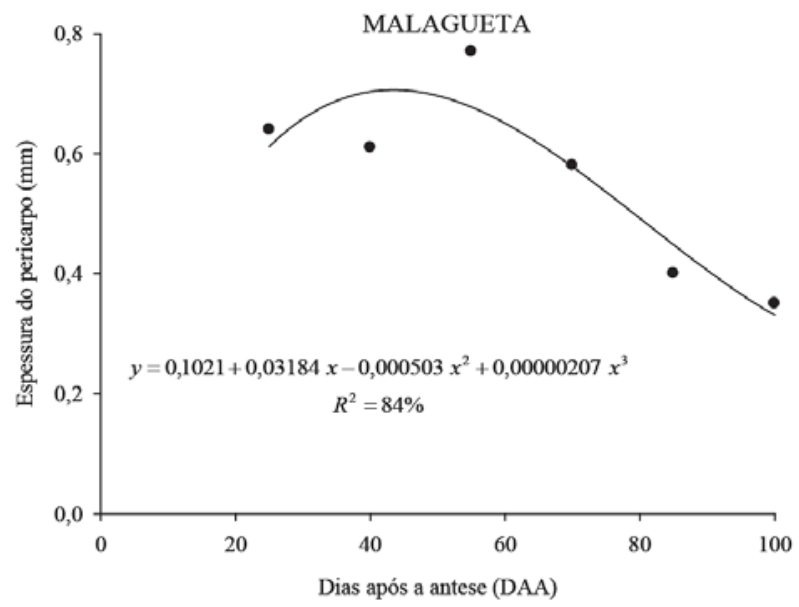

A

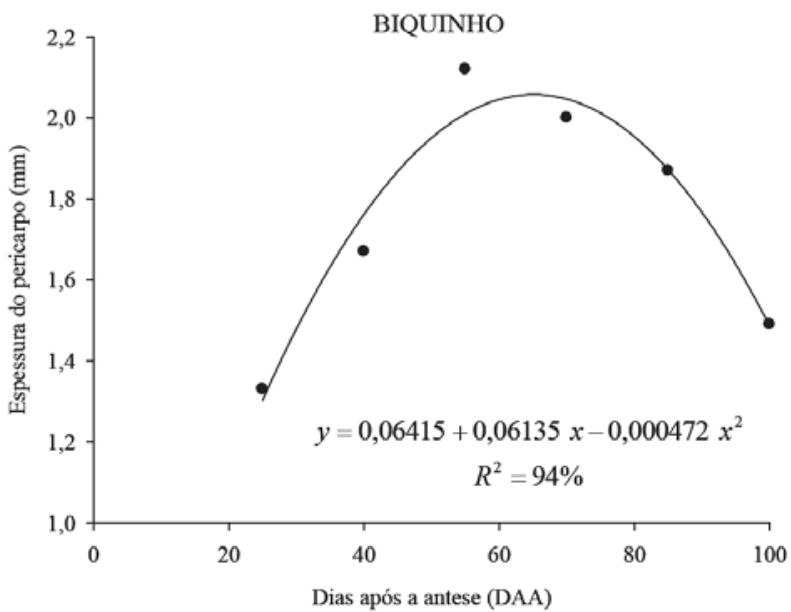

B

Figura 5 - Espessura do pericarpo dos frutos de pimenta-malagueta (A) e de pimenta-biquinho (B) em diferentes estádios de maturação.
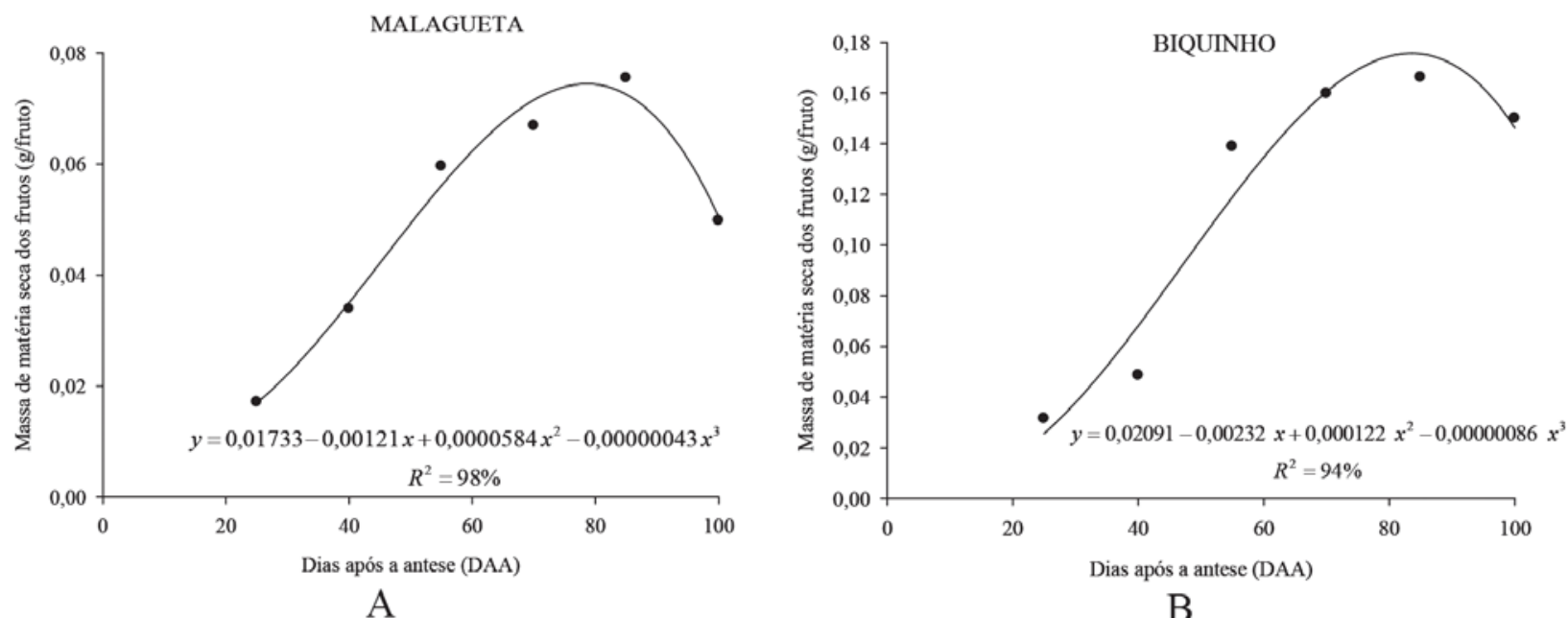

B

Figura 6 - Massa de matéria seca de frutos de pimenta-malagueta (A) e de pimenta-biquinho (B) em diferentes estádios de maturação.

malagueta. Houve aumento de massa até 79 DAA em que os frutos apresentavam em média $0,07421 \mathrm{~g}$ /fruto (Figura 6A). A partir deste período houve uma redução dos valores da massa dos frutos até $0,05033 \mathrm{~g}$ /fruto constatada aos 100 DAA.

Para a pimenta-biquinho, aos 25 DAA foram verificadas massa de $0,0257 \mathrm{~g} /$ fruto, havendo acréscimos de massa até 84 DAA, quando os frutos apresentavam em média $0,1771 \mathrm{~g} /$ frutos (Figura 6B). A partir desta época de maturação foram observados decréscimos nos valores desta variável, sendo que aos 100 DAA foi de $0,1489 \mathrm{~g} /$ fruto.

A redução nos valores das variáveis biométricas, em função da maturação de frutos carnosos, pode ser atribuída principalmente ao amolecimento do pericarpo, devido à ação de enzimas como a celulase e poligalacturonase que degradam a parede celular, realizam a hidrólise de amido e de outras macromoléculas, com consequente acúmulo de açúcares e redução nos 
teores de ácidos orgânicos, dentre outras alterações fisiológicas (Taiz \& Zeiger, 2009).

Além disso, durante a maturação dos frutos ocorre perda de água pela transpiração, sendo mais acentuada no fim deste processo, o que pode contribuir para a redução do peso dos frutos maduros. Peoples et al. (1985) afirmaram que, durante o processo de maturação dos frutos, inicialmente ocorre maior deposição de água, que é descarregada através do floema, quando comparada a água perdida durante o processo de transpiração. Posteriormente, os frutos perdem água tanto pela transpiração, que durante a secagem dos frutos é elevada, assim como ocorre uma recirculação de água do fruto para a planta por meio do xilema. A perda de água pelos frutos é responsável também por outras alterações tais como redução do brilho dos frutos, palatabilidade e qualidade nutricional.

Dias et al. (2006) também atribuíram a redução nos valores de algumas variáveis morfométricas, a partir de determinado estádio de maturação, de espécies de crescimento indeterminado, ao fato de que existe diferença na ordem de frutificação. Estes autores observaram que frutos de tomate colhidos de racimos mais tardios possuíam menor peso quando comparados àqueles colhidos no primeiro racimo.

Barbosa et al. (2010) avaliaram as características morfométricas dos frutos de diferentes espécies de pimentas Capsicum provenientes do estado de Roraima e verificaram para $C$. chinense o peso médio de 3,47 $\mathrm{g}$, comprimento médio de $3,62 \mathrm{~cm}$ e largura média de $1,63 \mathrm{~cm}$ e, para frutos de $C$. frutescens, $0,30 \mathrm{~g}$ de peso médio, $1,70 \mathrm{~cm}$ de comprimento e $0,48 \mathrm{~cm}$ de largura. Os autores relacionaram as características morfométricas dos frutos com a preferência da população pelos morfotipos de frutos ofertados e afirmaram que, em geral, são utilizados frutos de baixo peso, com alta ou baixa pungência, para molhos ou pimenta em pó. Os frutos de peso elevado, independente da sua pungência, são utilizados in natura em saladas. Aliyu \& Olarewaju (1994) afirmaram que os critérios de escolha do consumidor por frutos de pimenta baseia-se principalmente na consistência e dimensões morfológicas dos frutos, além da sua pungência.

Durante o processo de maturação dos frutos de pimenta foi possível verificar que as alterações fisiológicas, mensuradas por meio de características morfométricas, variou tanto entre as espécies quanto ao início das modifícações para cada variável. Houve acréscimo de valores até determinada época de maturação e a partir desse período, foram observadas reduções que, para a pimenta-malagueta, iniciaram a partir de 43 DAA para a espessura do pericarpo dos frutos. A massa de matéria seca foi a variável que apresentou reduções mais tardias, iniciando aos 79 DAA. Já para a pimenta-biquinho, houve maior uniformidade das épocas em que as reduções das variáveis morfométricas foram iniciadas em torno dos $65 \mathrm{DAA}$, exceto para o comprimento e massa de matéria seca.

\section{CONCLUSÕES}

Os frutos de pimenta-malagueta e de pimentabiquinho apresentam alterações de coloração no início do processo de maturação e alterações de turgescência após a maturidade fisiológica.

Existe correlação positiva entre as variáveis morfométricas massa, comprimento, diâmetro e espessura do pericarpo de frutos para as pimentas Malagueta e Biquinho.

Com a maturação dos frutos das pimentas Malagueta e Biquinho ocorrem acréscimos nos valores de variáveis morfométricas até determinada época de maturidade e, a partir de 43 DAA e 65 DAA, respectivamente, são observadas reduções.

\section{AGRADECIMENTOS}

ACAPES, CNPq e FAPEMIG pelo apoio financeiro à pesquisa.

\section{LITERATURA CITADA}

ALIYU, L.; OLAREWAJU, J.D. Variation in morphological and agronomic characters in sweet pepper (Capsicum annuum L.). Capsicum and Eggplant Newsletter, v.13, p.62-63, 1994.

BARBOSA, R.I.; MOURÃO JÚNIOR, M.; LUZ, F.J.F. Morphometric patterns and preferential uses of Capsicum peppers in the State of Roraima, Brazilian Amazonia. Horticultura

Brasileira, v.28, p.477-482, 2010.

BENTO, C.S.; SUDRÉ, C.P.; RODRIGUES, R.; RIVA, E.M.; PEREIRA, M.G. Descritores qualitativos e multicategóricos na estimativa da variabilidade fenotípica entre acessos de pimenta. Scientia Agraria, v. 8, p.149-156, 2007. 
BOSLAND, P.W.; COON. D.; REEVES, G. 'Trinidad Moruga Scorpion' Pepper is the N.4, World's Hottest Measured Chile Pepper at More Than Two Million Scoville Heat Units. Hort Technolog, v.22, n.4, p.534-538, 2012. Disponível em: http://horttech. ashspublications.org/content/22/4/ 534.full.pdf+html. Acesso em: jun.2017.

CEASAMINAS. Foram consultados os dados de 2016. Disponível em: http://minas2.ceasa.mg.gov.br/ detec/cst_prdvar_consolidado/ cst_prdvar_consolidado.php p. Acesso em: jul.2017.

DIAS, D.C.F.S.; RIBEIRO, F.P.; DIAS, L.A.S.; SILVA, D.J.H.; VIDIGAL, D.S. Tomato seed quality harvested from different trusses. Seed Science and Technology, v.34, p.681-689, 2006.

DOMENICO, C.I.; COUTINHO, J.P.; GODOY, H.T.; MELO, A.M.T. Caracterização agronômica e pungência em pimenta de cheiro. Horticultura Brasileira, v.30, n.3, p.466-472, 2012.

FAOSTAT. 2016. Food and Agriculture Organization of the United Nations. Disponivel em: http://www.factfish.com/statistic/ chillies $\% 20$ and $\% 20$ peppers $\% 2 \mathrm{C} \% 20$ green $\% 2 \mathrm{C} \%$ 20 production \%20quantity. Acesso em: jun.2017.

FONSECA, R.M.; LOPES, R.; BARROS ,W.S.; LOPES, M.T.G.; FERREIRA, F.M. Morphologic characterization and genetic diversity of Capsicum chinense Jacq. accessions along the upper Rio Negro - Amazonas. Crop Breeding and Applied Biotecnology, v. 8, p.187-194, 2008.

GELETA, L.F.; LABUSCHAGNE, M.T.; VILJOEN, C.D. Genetic variability in pepper (Capsicum annuum L.) estimated by morphological data and amplified fragment length polymorphism markers.

Biodiversity and Conservation, v.14, p.2361-2375, 2005.

JUNIOR e SILVA, W.C.; CARVALHO, S.I.C.; DUARTE, J.B. Identification of minimum descriptors for characterization of Capsicum spp. germplasm. Horticultura Brasileira, v. 31, n.2, p.190-202, 2013.
LANNES, S.D.; FINGER, F.L.; SCHUELTER, A.R.; CASALI, V.W.D. Growth and quality of Brazilian accessions of Capsicum chinense fruits. Scientia Horticulturae, v.112, p.266-270, 2007.

LUTZ, D.L.; FREITAS, S.C. Valor nutricional. In: RIBEIRO, C.S.C.; CARVALHO, S.I.C.; HENZ, G.P.; REIFSCHNEIDER, F.J.B. (eds). Pimentas Capsicum. Brasília: Embrapa Hortaliças, 2008. p.31-38.

MARUYA, A.K.; KUSHWAHA, M.L.; MARUYA, S.K; PANCHBHAIYA, M. Estimation of Per se performace of chilli (Capsicum annuum L.) genotypes for yield and quality traits. Journal of Pharmacognosy and Phytochemistry, v.6, n.1, p.333-335, 2017.

OYAMA, K.; HERNANDEZ-VERDUGO, S.; SANCHEZ, C.; GONZALEZ-RODRIGUEZ, A.; SANCHEZ-PEÑA, P.; GARZON-TIZNADO, J.A.; CASAS, A. Genetic structure of wild and domesticated populations of Capsicum annuum (Solanaceae) from northwestern Mexico analysed by RAPDs. Genetic Resources and Crop Evolution, v.53, p.553-562, 2006.

PEOPLES, M.B; PATE, J.S.; ATKINS, C.A.; MURRAY, D.R. Economy of water, carbon, and nitrogen in the developing cowpea fruit. Plant Physiology, v.77, n.1, p.142-147, 1985.

PEREIRA, P.F.M.; LEITE, M.L.G.; LIMA, L.C.; STEFANI, R. Evaluation of antioxidant potential of pepper sauce (Capsicum frutescens L.).

Cogent Food \& Agriculture, v.2, p.1-7, 2016. Disponivel em: http://www.tandfonline.com/ doi/full/10.1080/23311932.2015.1134381. Acesso em: jun.2017.

PINO, J.; SAURI-DUCH, E.; MARBOT, R. Changes in volatile compounds of Habanero chile pepper (Capsicum chinense Jack. Cv Habareno) at two ripening stages. Food Chemistry, v.94, p.394-398, 2006.

PINTO, C.M.F.; PINTO, C.L.O.; DONZELES, S.M.L. Pimenta Capsicum: Propriedades químicas, nutricionais, farmacológicas e medicinais e seu potencial para o agronegócio. Revista Brasileira de Agropecuária Sustentável (RBAS), v.3, n.2, p.108$120,2013$. 
QUEIROZ, L.A.F; PINHO, E.V.R.V.; OLIVEIRA, J.A.; FERREIRA, V.F.; CARVALHO, B.O.; BUENO, A.C.R. Época de colheita e secagem na qualidade de sementes de pimenta HabaneroYellow.

Revista Brasileira de Sementes, v.33, n.3, p.472-481, 2011.

RÊGO, E.R.; RÊGO, M.M.; FINGER, F.L.; CRUZ, C.D.; CASALI, V.W.D. A diallel study of yield components and fruit quality in chilli peppers (Capsicum baccatum). Euphytica, v.168, p.275287, 2009.

RÊGO, E.R.; RÊGO, M.M.; FINGER, F.L.; CRUZ, C.D.; CASALI, V.W.D. Phenotypic diversity, correlation and importance of variables for fruit quality and yield traits in Brazilian peppers (Capsicum baccatum). Genetic Resources and Crop Evolution, v.58, n.6, p.909-918, 2011.

SUDRÉ, C.P.; RODRIGUES, R.; RIVA, E.M.; KARASAWA, M.; AMARAL JÚNIOR, A.T. Divergência genética entre acessos de pimenta e pimentão utilizando técnicas multivariadas.

Horticultura Brasileira, v.23, n.1, p.22-27, 2005.
TADESSE, T.; HEWETT, E.W.; NICHOLS, M.A.; FISHER, KJ. Changes in physicochemical attributes of sweet pepper cv. Domino during fruit grown and development. Scientia

Horticulturae, v.93, p.91-103, 2002.

TAIZ, L.; ZEIGER, E. Fisiologia Vegetal.

4.ed. Porto Alegre: Artmed, 2009. 848p.

THUL, S.T.; LAL, R.K.; SHASANY, A.K.; DAROKAR, M.P.; GUPTA, A.K.; VERMA, R.K.; KHANUJA, S.P.S. Estimation of phenotypic divergence in a collection of Capsicum species for yield related traits. Euphytica, v.168, p.189196, 2009.

VIDIGAL, D.S.; DIAS, D.C.F.S.; VON PINHO, E.R.D.; DIAS, L.A.S. Sweet pepper seed quality and Lea-protein activity in relation to fruit maturation and post-harvest storage. Seed Science and Technology, v.37, n.1, p.192201, 2009.

VIDIGAL, D.S.; DIAS, D.C.F.S.; DIAS, L.A.S.; FINGER, F.L. Changes in seed quality during fruit maturation of sweet pepper. Scientia Agricola, v.68, n.5, p.535-539, 2011.

Recebido para publicação em 09/01/2018 e aprovado em 02/06/2018. 